\title{
INFLUENCE OF QUANTITY OF PRINCIPAL COMPONENT IN DISCRIMINATIVE FILTERING
}

\author{
Kenny V. dos Santos ${ }^{1}$,Luiz Eduardo S. e Silva ${ }^{2}$ and Waldir S. S. Junior ${ }^{3}$ \\ ${ }^{1}$ DEQ/PPGEE/CETELI, Federal University of Amazonas, \\ Manaus, Amazonas, Brazil \\ kennyvinentedufam.edu.br \\ ${ }^{2}$ PPGEE/CETELI, Federal University of Amazonas, Manaus, Amazonas, Brazil \\ luiz.edu.sales@gmail.com, pedrodonadio7@gmail.com, \\ ${ }^{3}$ DTEC/PPGEE/CETELI, Federal University of Amazonas, Manaus, Amazonas, \\ Brazil \\ waldirjr@ufam.edu.br
}

\begin{abstract}
Discriminative filtering is a pattern recognition technique which aim maximize the energy of output signal when a pattern is found. Looking improve the performance of filter response, was incorporated the principal component analysis in discriminative filters design. In this work, we investigate the influence of the quantity of principal components in the performance of discriminative filtering applied to a facial fiducial point detection system. We show that quantity of principal components directly affects the performance of the system, both in relation of true and false positives rate.
\end{abstract}

\section{KEYWORDS}

Pattern Recognition, Discriminative Filtering, Principal Component Analysis \& Fiducial Points Detection.

\section{INTRODUCTION}

Facial fiducial points detection can be understood as a pattern recognition problem. Currently, there are several approaches that attempt to solve this problem. In general, these approaches propose a system for fiducial points detection and try to solve the problem via pattern recognition techniques. Some of these systems can be viewed in [1], [3], [4], [5], [6].

The papers published in [2], [7] proposes a pattern recognition technique that uses linear filtering and can be applied to fiducial points detection. Recently, a robust filter was design to fiducial points detection [1]. Those filters, called Discriminative Filters with Principal Component Analysis (FD-PCA), are designed using the theory of principal component analysis [10]. In this approach, the filters are designed to detect the principal components of higher variance associated with patterns of interest. In a facial fiducial points detection system, the quantity of principal components used directly impacts the system performance.

In this paper, we propose investigate the influence of the quantity of principal components in the performance of a facial fiducial points detection system. The experimental procedure is performed

Dhinaharan Nagamalai et al. (Eds) : CSE, DBDM, CCNET, AIFL, SCOM, CICS, CSIP - 2014

pp. 01-10, 2014. (C) CS \& IT-CSCP 2014

DOI : $10.5121 /$ csit.2014.4401 
using 11 fiducial points from a subset of 503 images from the BioID database [15]. From the results obtained, we can determine the number of principal components thats make satisfactory the system performance, using as criterion for evaluating the true positives (TP) and false positives (FP) rate.

This paper as organized as follows: Section II presents a review of concepts associated of discriminative filtering with principal component analysis. Section III presents the proposal of this work, constituted by the facial fiducial points detection system and experiments and results. And finally, the conclusions are commented in Section IV.

\section{Discriminative Filtering With PrinCiPal Component ANalysis}

\subsection{Discriminative Filtering}

The goal in discriminative filtering method is design an optimal linear filter $\boldsymbol{\Theta}$ which detects a pattern of interest $\mathbf{U}$ existing in an evaluation signal $\mathbf{G}$. An important feature of this method is the fact that it uses signal filtering for detection. The metric used to evaluate the signal $\mathbf{C}$ is the $\mathrm{DSNR}_{2}$ expressed by:

$$
\operatorname{DSNR}_{2}\left(m_{0}, n_{0}\right)=\frac{c\left(m_{0}, n_{0}\right)^{2}}{\sum_{i=0}^{\mathrm{M}-1} \sum_{j=0}^{\mathrm{N}-1} c(i, j)^{2}-c\left(m_{0}, n_{0}\right)^{2}}
$$

In [7], the authors propose a closed-form solution using an impulse restoration approach, which can be obtained as follows: given an array $g(m, n)$ which contains the pattern of interest $u\left(m-m_{0}\right.$ ,$\left.n-n_{0}\right)$ located at position $\left(m_{0}, n_{0}\right)$, and other signals that can be interpreted as an additive noise $b(m, n)$. Thus, we have:

$$
g(m, n)=u(m, n) * \delta\left(m-m_{0}, n-n_{0}\right)+b(m, n)
$$

Developing the Equation (2) and using the matrix notation, we can find the formulation of the impulse restoration problem as follows: given the signal $\mathbf{g}$ and an array $\mathbf{F}$ with dimensions $\mathrm{N} \times \mathrm{N}$, we must to find the best linear estimative of the vector $\hat{\boldsymbol{\delta}}=\mathbf{A g}$. Considering the case where the noise $\mathbf{b}$ is gaussian, with zero mean and covariance matrix equal to $\frac{\mathbf{1}}{\mathbf{N}} \mathbf{C}_{\mathbf{b}} 1 \mathrm{NC}$ b with dimensions $\mathrm{N} \times \mathrm{N}$, the vector $\hat{\boldsymbol{\delta}}$ shall be expressed by:

$$
\widehat{\delta}=\mathbf{F}^{T}\left[\mathbf{F F}{ }^{T}+\mathbf{C}_{\mathrm{b}}\right]^{-1} \mathbf{g}
$$

where the superscript ${ }^{\mathrm{T}}$ is the Hermitian. The discriminative filter $\Theta$ can be obtained by inspection of the linear estimator $\mathbf{A}$ [7].

\subsection{Discriminative Filtering with Principal Components Analysis}

In [1], the authors suggested the design of robust discriminative filters. In this approach, the discriminative filters are designed using principal components [10] of the set of matrices formed by occurrences of the pattern of interest. Mathematically, we can obtain the discriminative filters 
as follows: suppose a random variable $U_{N x I}$ with $M$ realizations equal to the vectors $\mathbf{u}_{\mathbf{1}}, \ldots, \mathbf{u}_{\mathbf{M}}$. The principal components $\boldsymbol{\Phi}=\left[\phi_{1}, \ldots, \phi_{N}\right]$ and their eigenvalues $\lambda_{1}, \ldots, \lambda_{\mathrm{N}}$ can be obtained using the solution of the eigenvalues problem [10] described below:

$$
\Lambda=\Phi^{T} \Sigma_{\mathcal{U}} \Phi,
$$

where $\Sigma_{U}$ is the covariance matrix from $U$.

The two-dimensional discriminative filters $\boldsymbol{\Theta} \phi_{1}, \ldots, \boldsymbol{\Theta} \phi_{S}$ are designed for $S$ principal components $\phi_{1}, \ldots, \phi_{S}$ with associated eigenvalues $\lambda_{1}, \ldots, \lambda_{\mathrm{s}}$, according the Equation (3). Thus, the equation of the estimator is given by:

$$
\mathbf{A}_{\phi_{i}}=\mathbf{F}_{\phi_{i}}^{H}\left[\mathbf{F}_{\phi_{i}} \mathbf{F}_{\phi_{i}}^{H}+\mathbf{C}_{\mathbf{b}_{i}}\right]^{-1} .
$$

Finally, the authors interpret $\mathbf{C}_{\mathbf{b i}}$ how an orthogonal subspace from the principal component of interest. In this case, the covariance matrix $\mathbf{C}_{\mathbf{b i}}$ associated with $\phi_{\mathrm{i}}$ can be written as:

$$
\mathbf{C}_{\mathbf{b} i}=\sum_{\substack{j=1 \\ j \neq i}}^{N} \psi_{j} \mathbf{F}_{\phi_{j}} \mathbf{F}_{\phi_{j}}^{H},
$$

where $\psi_{j}$ are constants that indicate the statistical noise, and $\mathrm{F} \phi_{\mathrm{j}}$ is the circular matrix by blocks obtained from the component $\phi_{\mathrm{j}}$.

\section{EXPERIMENTS AND RESULTS}

\subsection{Facial Fiducial Points Detection System}

The performance evaluation of robust discriminative filters was performed using a supervised facial fiducial points detection system. This system has two stages: training and test (Figures 1 and 2). In both cases, we have a pre-processing block for the images. This block has the configuration presented in [1]: a Viola-Jones face detector [11], a scaling block to $220 \times 220$ resolution, an illumination correction block [12] and a Gaussian Prior Model [1].

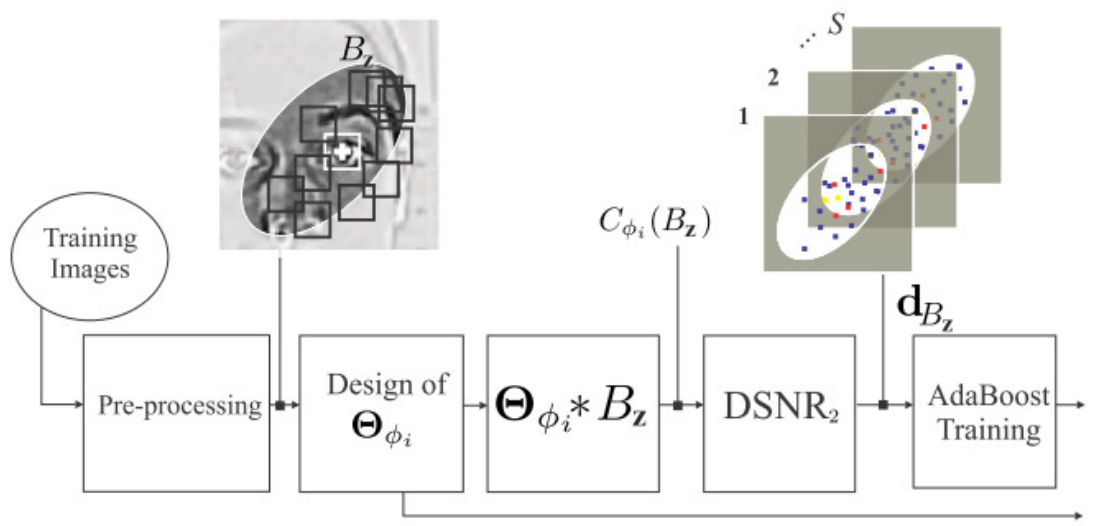

Figure 1. Training procedure of the fiducial points detection system. 


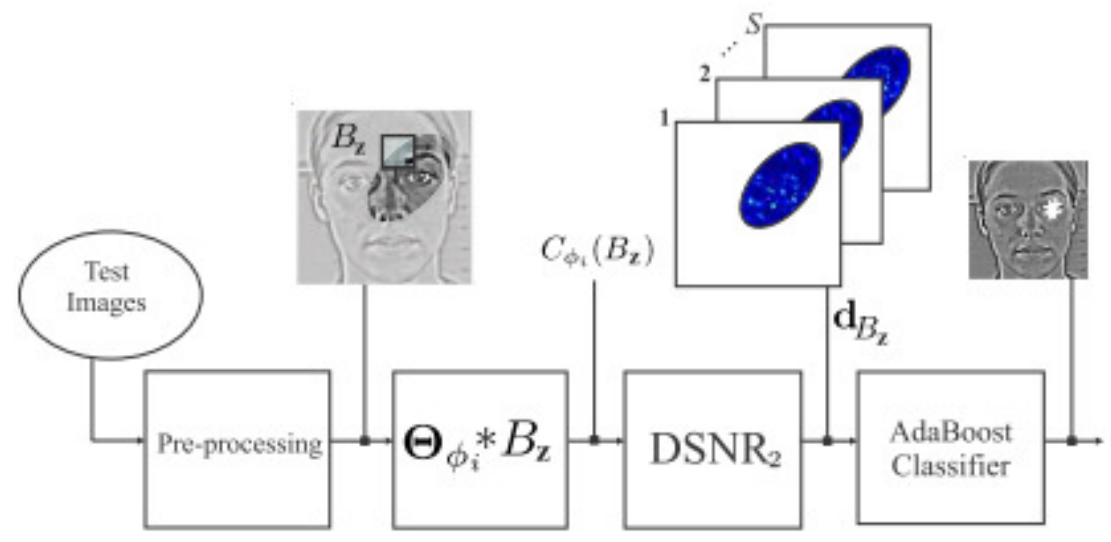

Figure 2. Training procedure of the fiducial points detection system.

The training procedure (Figure 1) can be described as follows: first, we perform a pre-processing at the image. Then, the $S$ discriminative filters $\boldsymbol{\Theta} \phi_{\mathrm{i}}$ will be designed for each of the principal components. Through a sliding window, each $B_{z}$ block belonging to the elliptical region of interest is filtering by all $S$ filters $\Theta \phi_{\mathrm{i}}$, generating $S$ matrices $\mathrm{C} \phi_{\mathrm{i}}\left(B_{z}\right)$. Using the Equation (1) we obtain the $\mathrm{DSNR}_{2}$ values of the matrices $\mathrm{C} \phi_{\mathrm{i}}\left(B_{z}\right)$. So, each block $B_{z}$ has a $\mathrm{DSNR}_{2}$ associated vector named $\mathbf{d}_{\boldsymbol{B} z}$ with dimensions $1 \times S$. The vectors $\mathbf{d}_{\boldsymbol{B} z}$ will be used for training of the AdaBoost classifier using the GML AdaBoost toolbox [14]. The output of the training stage consist of $\Theta \phi_{\mathrm{i}}$ filters, the AdaBoost classifier and the mean block of the patterns of interest, called $\boldsymbol{\mu}_{U}$.

The test procedure, presented at Figure 2, can be described by: first, the input image is preprocessed. Then, using a sliding window, we process each $B_{z}$. block. The matrices $C \phi_{\mathrm{i}}\left(B_{z}\right)$. are obtained by filtering between $B_{z}$, subtracting by $\boldsymbol{\mu}_{U}$ and each of the $S$ filters $\boldsymbol{\Theta} \phi_{\mathrm{i}}$. After, we calculate the $\mathrm{DSNR}_{2}$ for each $\mathrm{C} \phi_{\mathrm{i}}\left(B_{z}\right)$, resulting in a $\mathrm{DSNR}_{2}$ associated vector named $\mathbf{d}_{\boldsymbol{B} z}$. The AdaBoost classifier will categorized $\mathbf{d}_{B z}$ as positive (the center of $B_{z}$ is the fiducial point) or negative (the center of $B_{z}$ isn't the fiducial point).

\subsection{Experiments and Results}

To evaluate the effect of the quantity of principal components in the facial fiducial points detection system which uses discriminative filters, we use a total of 11 fiducial points and a subset of 503 images from the BioID database [15]. The fiducial points and your numeration are presented at the Figure 3. In all experiments, we use cross-validation with 7 folds [13]. We used 6/7 of the total images and use in the training step and 1/7 for the test step. For this experiment, we varied the quantity of principal components $(S)$ used in the proposed system as follows: $S=$ $[8,13,23,33,43,53,63,73,83,93,100]$.

The system's performance is measured using the intraocular distance. This distance, designated $\tilde{d}_{o}$, is obtained as follows:

$$
\widetilde{d}_{o}=\|\widetilde{\mathbf{O E}}-\widetilde{\mathbf{O D}}\|
$$

where: $\widetilde{\mathrm{OE}}$ corresponds to the coordinates of the manual label from the left pupil and $\widetilde{\mathrm{OD}}$ are the coordinates of the manual label from the right pupil. For validation, we use the true and false positive rates, presented in [1]. We consider a candidate of fiducial point any mark which have distance from the manual fiducial point less then $10 \% \tilde{d}_{o}$. 
Figures 4 to 9 show the results obtained as a function of the quantity of principal components for fiducial points $0,1,2,6,7$ and 9 . The blue curves relate the true positives rate and the red curve relating the false positives rate. Due to the symmetry of the face, we present only the curves of the fiducial points located at the left side of the face. For comparison, the best results are summarized in Table I. In this table, we compared the best results of our proposed method with the state-of-art method Support Vector Machines. To perform this comparison, we performed the experiments using two approaches: the linear SVM (SVM-L) and polynomial SVM (SVM-P) [8], [9].

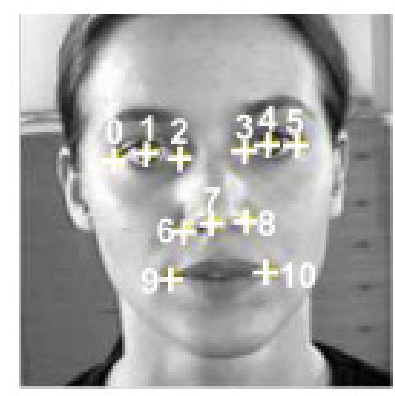

Figure 3.Fiducial points used in the experiments.

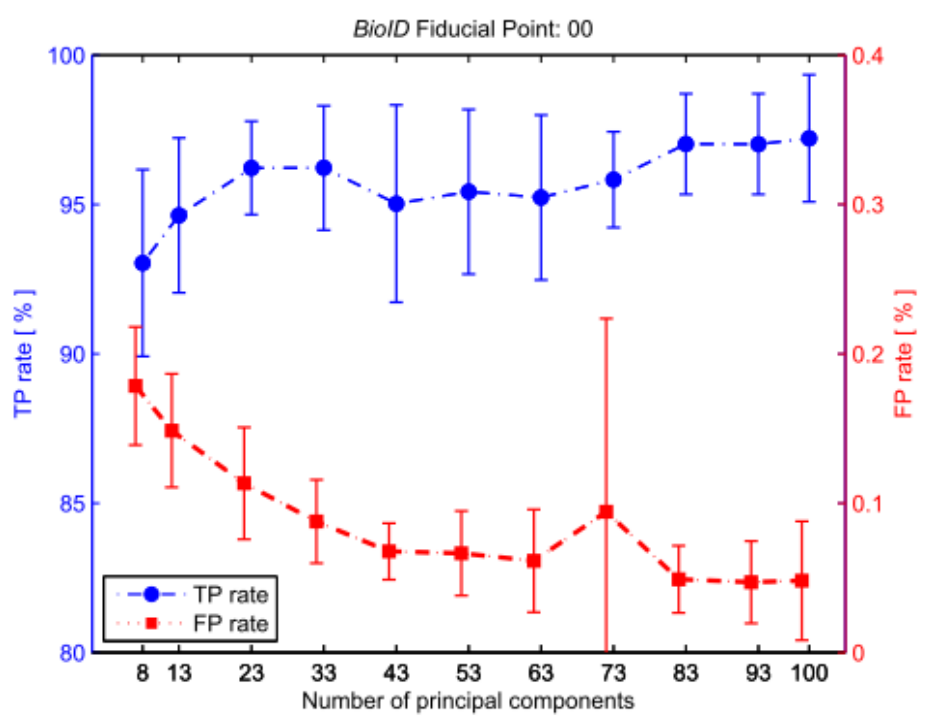

Figure 4. TP and FP curves for fiducial point 00. 


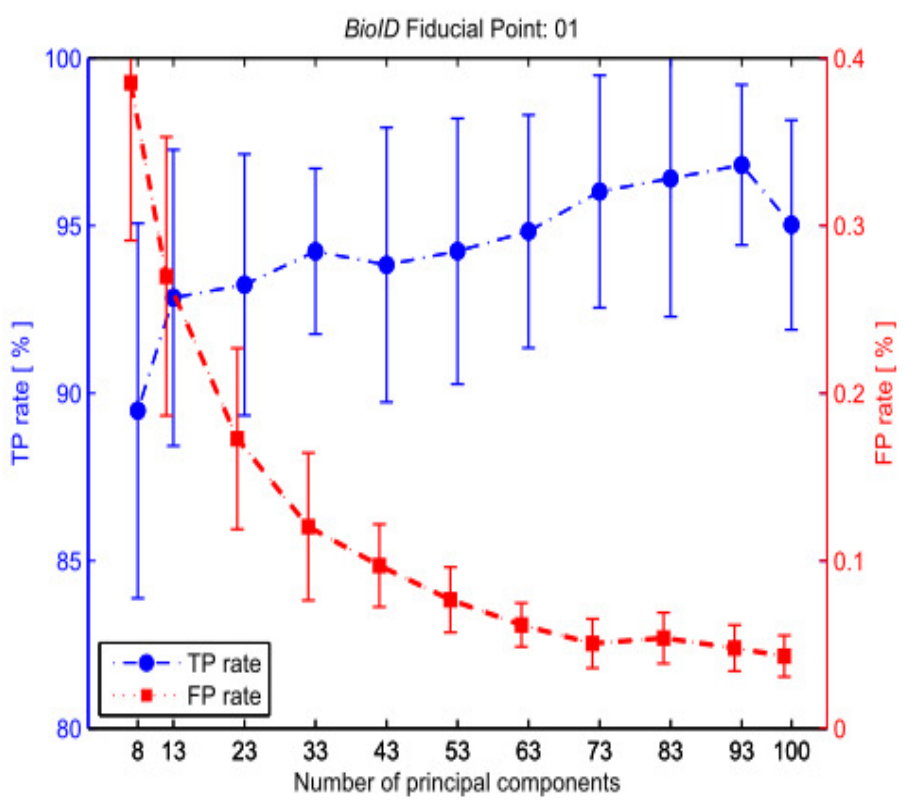

Figure 5. TP and FP curves for fiducial point 01.

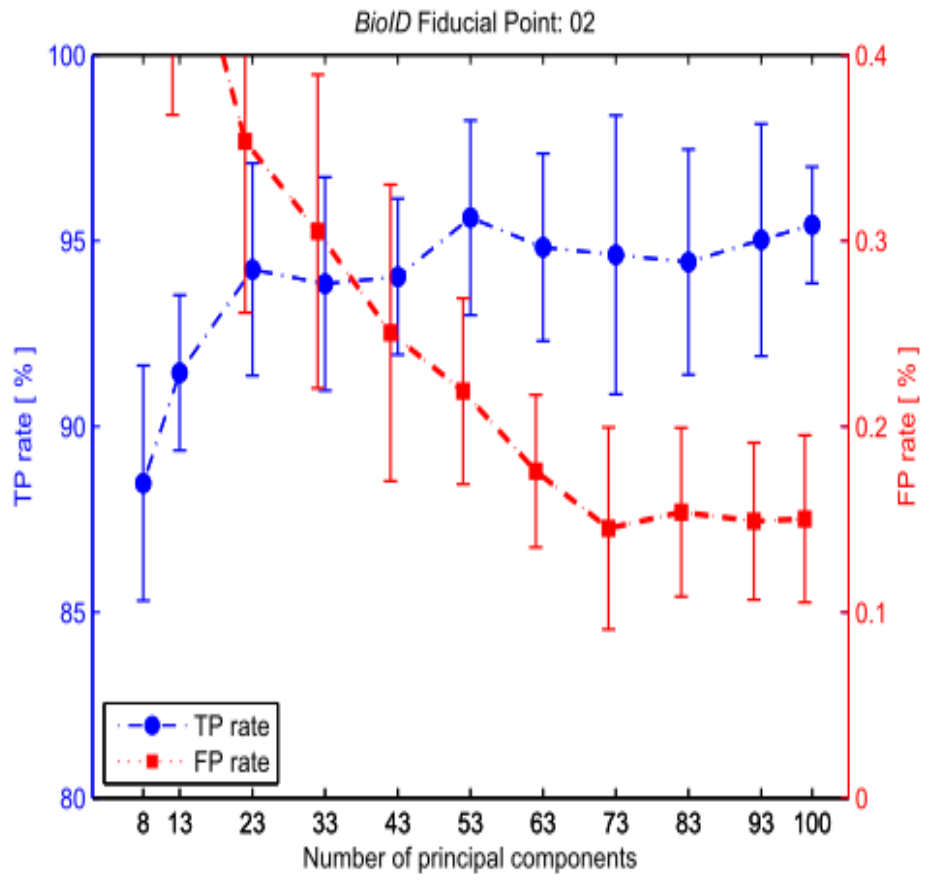

Figure 6. TP and FP curves for fiducial point 02. 


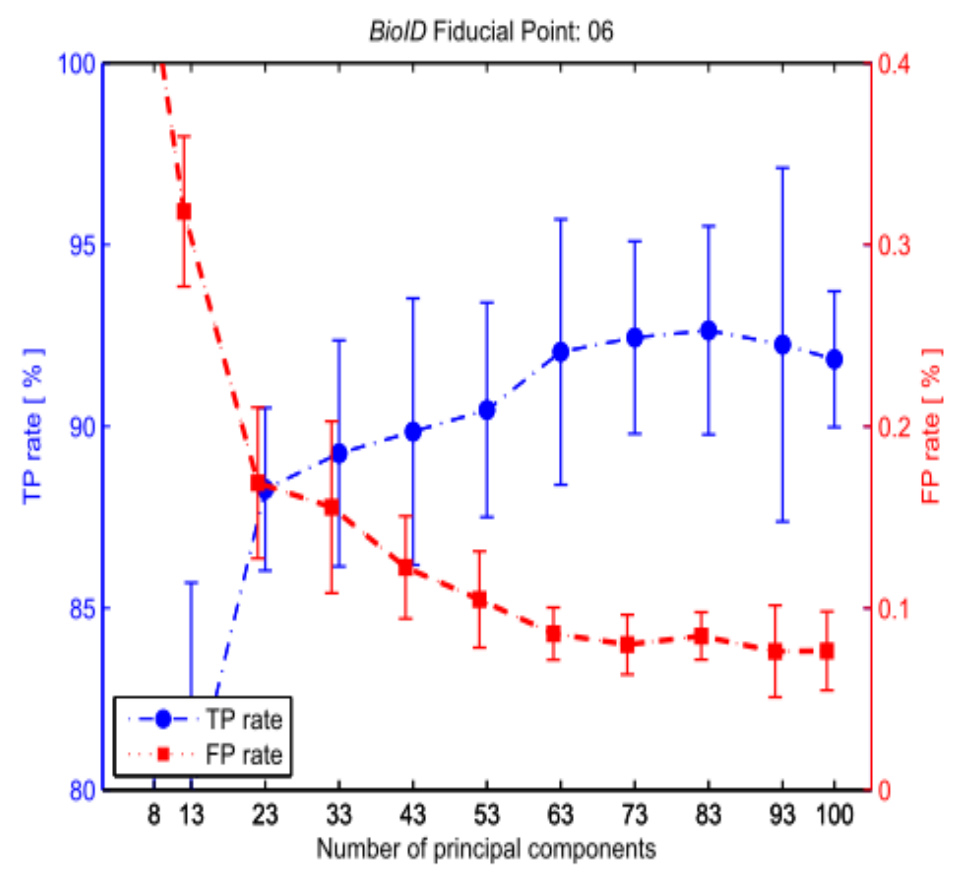

Figure 7. TP and FP curves for fiducial point 06.

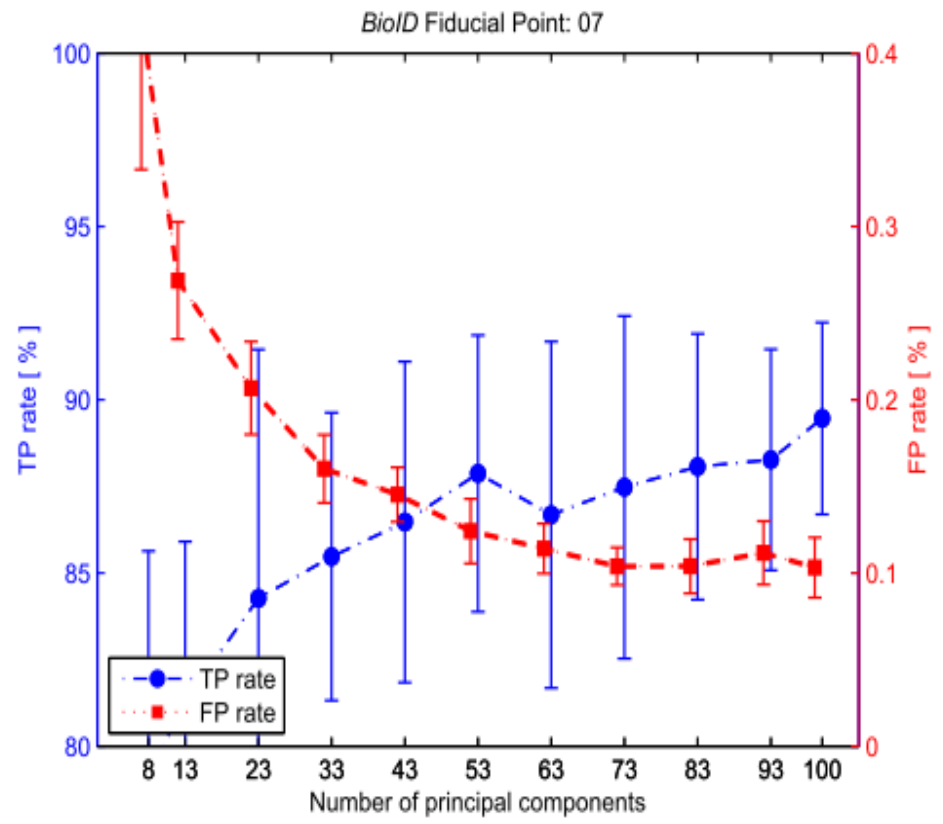

Figure 8. TP and FP curves for fiducial point 07. 


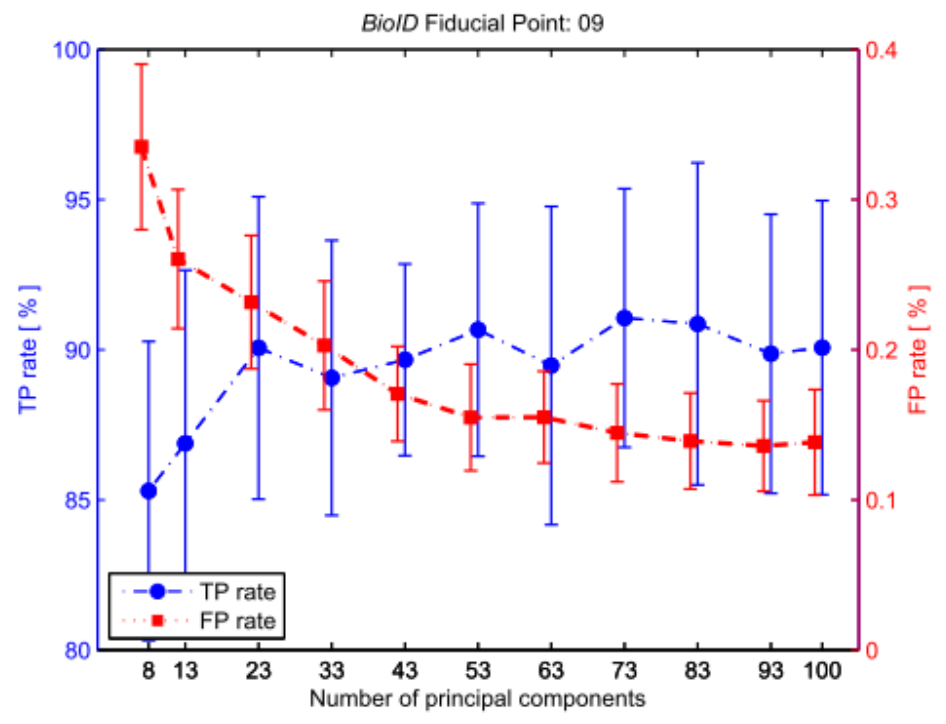

Figure 9. TP and FP curves for fiducial point 09.

From the curves presented at Figures 4 to 9, we can conclude that increasing the quantity of principal components used improves the system performance. In relation to Table I, we find that proposed method is better than SVM-L at the fiducial points 0 and 2 . We also observed that for the most of points, our method is slightly lower than SVM-L and SVM-P in terms of true positives rate and superior in terms of false positives rate.

Table I. Results obtained to 11 fiducial points from BioID database.

\begin{tabular}{|c|c|c|c|c|c|c|c|c|c|c|c|c|c|}
\hline \multirow[t]{2}{*}{ Fiducial Point } & \multicolumn{5}{|c|}{$\begin{array}{l}\text { FD-PCA } \\
\text { (proposed) }\end{array}$} & \multicolumn{4}{|c|}{ SVM-L } & \multicolumn{4}{|c|}{ SVM-P } \\
\hline & TP & $\sigma$ & FP & $\sigma$ & $\mathrm{NC}$ & TP & $\sigma$ & FP & $\sigma$ & TP & $\sigma$ & FP & $\sigma$ \\
\hline 0 & 97,0 & 1,68 & 0,05 & 0,02 & 83 & 92,4 & 9,8 & 1,09 & 0,74 & 99,6 & 0,7 & 0,39 & 0,07 \\
\hline 1 & 96,8 & 2,39 & 0,05 & 0,01 & 93 & 99,6 & 1,0 & 0,32 & 0,09 & 98,8 & 1,2 & 0,04 & 0,02 \\
\hline 2 & 95,4 & 1,57 & 0,15 & 0,04 & 100 & 93,0 & 14,3 & 1,58 & 0,59 & 94,2 & 2,7 & 0,15 & 0,03 \\
\hline 3 & 95,4 & 2,07 & 0,13 & 0,03 & 93 & 96,0 & 1,7 & 0,46 & 0,21 & 99,8 & 0,5 & 0,33 & 0,09 \\
\hline 4 & 96,0 & 0,96 & 0,05 & 0,02 & 100 & 97,2 & 4,2 & 2,49 & 4,76 & 98,8 & 1,5 & 0,08 & 0,05 \\
\hline 5 & 96,8 & 2,36 & 0,03 & 0,01 & 100 & 98,8 & 3,1 & 2,27 & 0,62 & 99,6 & 0,7 & 0,42 & 0,09 \\
\hline 6 & 92,6 & 2,86 & 0,08 & 0,01 & 83 & 99,6 & 1,0 & 1,40 & 1,44 & 99,6 & 0,7 & 0,31 & 0,05 \\
\hline 7 & 89,4 & 2,76 & 0,10 & 0,01 & 100 & 47,1 & 9,6 & 0,06 & 0,03 & 99,6 & 0,7 & 0,60 & 0,05 \\
\hline 8 & 93,6 & 2,63 & 0,09 & 0,01 & 93 & 95,4 & 3,9 & 0,40 & 0,09 & 99,0 & 1,1 & 0,24 & 0,03 \\
\hline 9 & 91,0 & 4,30 & 0,14 & 0,03 & 73 & 96,8 & 5,5 & 2,91 & 5,12 & 98,8 & 1,7 & 0,39 & 0,06 \\
\hline 10 & 85,8 & 4,80 & 0,07 & 0,04 & 63 & 98,6 & 1,9 & 1,47 & 0,49 & 99,6 & 0,7 & 0,35 & 0,04 \\
\hline
\end{tabular}

\section{CONCLuSiON}

In this work we investigated the influence of the quantity of principal components in the performance of a facial fiducial points detection system. We performed the experiments in the BioID database, using cross-validation, splitting the total of images in two segments: training set (6/7 of total) and test set (1/7 of total). In this experiment, we varied the quantity of principal components and studied the influence of this quantity of components in the system performance, using as criteria the true and false positives rate. The results showed that quantity of principal components used determine the system performance. A low number of components will generate underperforming systems. We also observed that as of 43 components we obtain systems with 
good performance and from 73 components the false positives rate stabilizes. Finally, we conclude that the proposed method can compete with the state-of-art method SVM.

\section{ACKNOWLEDGEMENT}

We would like to thanks Samsung for financial support. This research was supported by FAPEAM and CAPES agency.

\section{REFERENCES}

[1] W. S. da Silva Júnior, G. M. Araújo, E. A. B. da Silva and S. K. Goldenstein, "Facial Fiducial Points Detection Using Discriminative Filtering on Principal Components". In: Proceedings of the IEEE International Conference on Image Processing, September, pp. 2681-2684, 2010.

[2] A. P. Mendonça, E. A. B. Silva, "Two-Dimensional Discriminative Filters for Image Template Detection" In: Proceedings of the International Conference on Image Processing, pp. 680-683, 2001.

[3] B. Martinez, M. F. Valstar, X. Binefa, M. Pantic, "Local Evidence Aggregation for Regression Based Facial Point Detection" In: IEEE Transactions on Pattern Analysis and Machine Intelligence, pp. $1149-1163,2013$.

[4] F. Jerome, H. Trevor and R. Tibshirani, "Additive Logistic Regression: A Statistical View of Boosting", 'In: Annals of Statistics, vol. 28, pp. 2000, 1998.

[5] T. Cootes, G. J. Edwards and C. J. Taylor, "Active Appearance Models", 'In: Proceedings of the European Conference on Computer Vision, vol.2, pp. 484-498, 1998.

[6] G. M. Araujo, W. S. da Silva Júnior, E. A. B. da Silva, and S. K. Goldenstein, "Facial Landmarks Detection based on Correlation Filter", In: Proceedings of the IEEE International Telecommunication Symposium, Manaus, AM, Brazil, October 2010.

[7] A. P. Mendonça, E. A. B. Silva, "Closed-Form Solutions for Discriminative Filtering using Impulse Restoration Techniques", IEE Electronics Letters, vol. 38, n. 22, pp. 1332-1333, 2002.

[8] D. Wu, F. Cao, "Learning rates for SVM classifiers with polynomial kernels". In: Proceedings of the Eighth International Conference on Machine Learning and Cybernetics, Baoding, July, 2009.

[9] G. L. Prajapati, A. Patle, "On Performing Classification Using SVM with Radial Basis and Polynomial Kernel Functions". In: Third International Conference on Emerging Trends in Engineering and Technology, 2010.

[10] M. Kirby and L. Sirovich, "Application of the Karhunen-Loève Procedure for the Characterization of Human Faces", IEEE Transactions on Pattern Analysis and Machine Intelligence, vol. 12, 1990.

[11] Viola, P. and Jones, M., "Robust Real-Time Object Detection". International Journal of Computer Vision, vol. 57, n. 2, pp. 137-154, 2001.

[12] Xiaoyang, T. and Triggs, B., Enhanced Local Texture Feature Sets for Face Recognition Under Difficult Lighting Conditions. IEEE Transactions on Image Processing, vol. 19, n. 6, pp. 1635-1650, 2010.

[13] Kohavi, R., "A Study of Cross-Validation and Bootstrap for Accuracy Estimation and Model Selection", In: Proceedings of the International Joint Conference on Artificial Intelligence, August, pp. 1137-1143, 1996.

[14] "The GML AdaBoost Matlab Toolbox", [Last access in August 2013]. [Online]. Avaliable in: http://graphics.cs.msu.ru/en/science/research/machinelearning/adaboosttoolbox .

[15] "Bioid Database", [Last access in October 2013]. [Online]. Avaliable in: http://www.bioid.com/ 


\section{Authors}

Kenny V. Santos is a graduate student in Electrical Engineering from Federal University of Amazonas. He received his bacharel degree in Electrical Engineering at Federal University of Amazonas in 2008. Currently he works in the fields of computer vision and pattern recognition, where his main interest is applications of digital signal processing in pattern recognition.

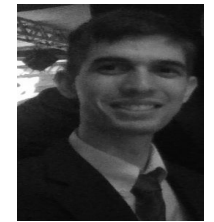

Luiz Eduardo S. e Silva received the B.S. degree in electrical engineering at the Federal University of Amazonas (UFAM), Manaus, AM, Brazil, in 2011. Currently, he is a M.Sc. student at the same University and he is working with computer vision. Support Vector Machine is a technique applied in his pattern recognition researches, which try to classify elements of interest. He is Professor of Nokia Foundation in Electrical Circuits e Telecommunications. Automatic Control, Digital Signal Processing, Machine Learning and Mathematical Morphology are also his interest areas.

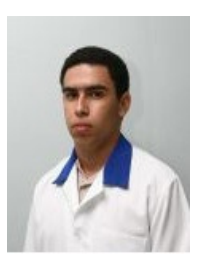

Waldir S. S. Júnior received the B.S. degree in electrical engineering at the Federal University of Amazonas (UFAM), Manaus, AM, Brazil, in 2000, and the M.S. degree in electrical engineering at the Federal University of Rio de Janeiro (COPPE/UFRJ), Rio de Janeiro, RJ, Brazil, in 2004 and Ph.D. degree in electrical engineering at the Federal University of Rio de Janeiro (COPPE/UFRJ), Rio de Janeiro, RJ, Brazil, in 2010. Since 2006, he has been with the Federal University of Amazonas, as Full Professor. His research interests are in the fields of data compression, as well as in mathematical morphology, pattern recognition and digital signal processing in general.

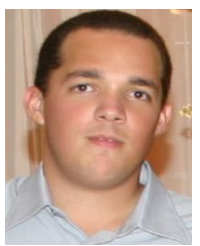

